\title{
An EMD and IMF Energy Entropy Based Optimized Feature Extraction and Classification Scheme for Single Trial EEG Signal
}

\section{Song Luo}

Guizhou Normal University

\section{PeiYun Zhong}

Guizhou Normal University

\section{Rui Chen}

Guizhou Normal University

CunYang Pan

Guizhou Normal University

KeYu Liu

Guizhou Normal University

Kun Li ( $\nabla$ kunli@gznu.edu.cn )

Guizhou Normal University

\section{Research Article}

Keywords: BCl, Improved EMD scheme, IMF energy entropy, SVM, voting mechanism

Posted Date: December 29th, 2021

DOI: https://doi.org/10.21203/rs.3.rs-1161236/v1

License: () (1) This work is licensed under a Creative Commons Attribution 4.0 International License. Read Full License 


\section{Abstract}

For the purpose of improving the classification accuracy of single trial EEG signal during motor imagery (MI) process, this study proposed a classification method which combined IMF energy entropy and improved EMD scheme. Singular value decomposition (SVD), Gaussian mixture model, EMD and IMF energy entropy were employed for the newly designed scheme. After removing noise and artifacts from acquired EEG signals in EEGLAB, SVD was applied, and the singular values were clustered by Gaussian mixture model. The insignificant characteristics indicated by the small SVD values were then removed, and the signals were reconstructed, feeding to EMD algorithm. Those IMFs mapping to $\delta \square \theta \square a$ and $\beta$ frequencies were selected as the major features of the EEG signal. The SVM classifier with RBF, linear, and polynomial kernel functions and voting mechanism then kicked in for classification. The results were compared with that of the traditional EMD and EEMD through simulation, showing that the proposed scheme can eliminate mode mixing effectively and improve the single trial EEG signal classification accuracy significantly, suggesting the probability of designing a more efficient EEG control system based on the proposed scheme.

\section{Introduction}

The functions and working mechanisms of the human brain have attracted inexhaustible research interests. The U.S., EU, Japan and China started "Brain Plan" one after another, expecting to make breakthroughs in the field of brain research, thus opening a gate to an exciting future full of opportunities and challenges [1-4]. Brain computer interface (BCl), as a communication channel established between human brain and external environment, provides researchers a new perspective and a useful tool for the study of neural activity. Among all the $\mathrm{BCl}$ designs, event-related potential (ERP) based $\mathrm{BCl}$ design has been proved to be an effective strategy, which enables a subject to control external equipment via EEG signal produced in the process of "speculating", "perceiving", or "task implementing" [5]. Nonetheless, the low spatial resolution and signal to noise ratio of EEG signals make them easily contaminated by artifacts (such as EOG, EKG, EMG, and power-line interference), thus limiting the use of BCls [6]. Improving the classification accuracy of EEG signals, especially for the single trial EEG signals, has become a serious challenge in the field. In view of this, the present study focused attention on the improvement of single trial EEG signal classification accuracy.

Feature extraction is of great significance for brain wave processing and classification. EEG features in time domain, such as profile, standard deviation, correlation, peak value, etc., can be acquired directly, while in frequency domain, the features can be extracted by signal processing method, such as Fourier transform, power spectrum analysis, modern spectral estimation, coherence analysis, etc. However, most of the feature extraction methods base on an assumption that the acquired signals are linear and stationary, which is not suitable for EEG signal analysis. The critical information contained in EEG signals are most likely non-linear and non-stationary. Short-Time Fourier Transform, Gabor Transform, and Wavelet Transform, as they designed for non-linear stationary signal processing, partially resolved this issue [7]. Huang et al. proposed an adaptive signal processing method, Hilbert-Huang Transform (HHT), 
which based on Empirical Mode Decomposition (EMD) and Hilbert spectrum analysis, providing a better option for non-linear and non-stationary signal processing [8]. HHT has been applied by many researchers and achieved good results. For instance, Kevriv et al. achieved 92.8\% classification accuracy via combining EMD, discrete Wavelet Transform, and Wavelet packet processing techniques [9]; Taran et al. obtained $90 \%$ classification accuracy by analyzing the features of intrinsic mode functions (IMF) [10]; Tang et al. improved EEG classification accuracy via conditional EMD based one-dimensional and multidimensional convolution neural network and realized EEG based wheelchair control [11]. Previous studies have found that the $a$ and $\beta$ rhythms are suppressed in the process of motor imagery, while $\delta$ and $\theta$ rhythms experiencing time and phase locks, making the EEG signals non-linear and non-stationary [12]. Therefore, the present study used EMD to decompose EEG signal. As for entropy, it is a concept originally came from thermodynamics. Shannon employed it in his information theory and defined it as a measurement of the uncertainty of the source signal. Information entropy has been widely used in many research fields, such as pathological speech quality estimation, recognition fatigue measurement, dysphagia sound classification [13-15], and EEG based epilepsy diagnosis. These facts inspired us to combine EMD and information entropy in our research. And concurrently we have to deal with the mode mixing issue born with EMD. Although Ensemble Empirical Mode Decomposition (EEMD) proposes a possible way to solve the mode mixing issue[16], it requires the addition of white noise and the decomposition quality is determined by the number of times of averaging, making it less effective.

Tremendous researches have shown that the causes of mode mixing include intermittent signal, pulse interference and noise $[17,18]$. Reducing the influences of these factors will improve SNR and present us with much clearer signal features. Singular Value Decomposition (SVD), as it decomposes the signal matrix and assign each vector a singular value, may help us to extract critical features of the signal according to the singular values [19]. The size and variation of the singular values vary with different signals. Inappropriate selection of them may cause information loss or noise increasing, thus a wise selection of singular values is the key to successful classification. Traditionally, singular value curve is employed to fulfill the task, yet this method usually depends on the experience of the operator. K-means clustering, another option for singular value selection, is easily affected by the initial data center, thus lacking robustness [20]. Gaussian Mixture Model (GMM) uses a combination of multiple Gaussian distributions to describe data distribution, adaptively optimizing clustering [21,22], making it a more suitable option for non-linear and non-stationary signal classification scheme design. After EMD decomposition, the energy entropies $[23,24]$ of intrinsic mode functions mapping to $d, q$, a and $b$ frequencies will be used as the feature set. In this work, SVD, GMM, EMD and energy entropy were combined to improve the feature extraction performance. The extracted features were then fed to SVM classifiers, which give us the final results through their voting [25-28].

Comparing with that of the traditional EMD and EEMD, our results showed that the proposed scheme could eliminate mode mixing more effectively; energy entropies of the IMFs were suitable features for the classification of EEG signals generated in the process of $\mathrm{Ml}$; voting was a validate mechanism significantly improving classification accuracy and robustness. It is worth mentioning that all the methods and results we discussed above were regarding to single trial EEG signal classification. 


\section{Materials And Methods}

All the methods and protocols were carried out in accordance with relevant guidelines and regulations and approved by Guizhou Normal University medical ethic committee. Informed consents were obtained from all subjects.

\section{1 $\mathrm{BCl}$ design}

In this work, an Oddball paradigm[29] based BCI was designed, for which a rare event will elicit P300 response, an event-related potential corresponding to target stimulation. Five pictures indicating five directions (up, down, left, right, and stop) were used as visual stimuli. Each stimulation continued for 125 $\mathrm{ms}$, followed by a $600 \mathrm{~ms}$ blank interval. A single trial consists of five stimuli of different directions in random order, one of which would be the target stimulation, eliciting P300 response. The subject was asked to press down corresponding keys on the numpad to mark the target stimulation (the five directions were mapping to the numpad; up, down, left, right, and stop for number $8,2,4,6$, and 5 , respectively). A total of 100 trials were recorded via Neuroscan system. The designed $\mathrm{BCl}$ was programed in E-prime. Figure 1. Showed the stimulation sequence and the time windows.

\subsection{Data collection}

32 healthy people (16 males and 16 females) aged between 19-23, without neurological diseases and disabilities, were recruited as our subjects. The subjects were asked sitting in front of a screen, relaxing themselves, avoiding body movements, reducing eye blinks, and focusing their attention on the center of the screen. The $\mathrm{BCl}$ designed by us keep presenting direction pictures to the subjects. Once an intended direction shown on the screen, the subjects pressed down a corresponding key to mark it. The EEG signals were recorded in Neuroscan system. Since P300 response usually occur at the parietal occipital region of the scalp, 10 channels around this area (F3, FZ, F4, C3, CZ, C4, P3, PZ, P4, and OZ) were selected for data collection. The sampling frequency was set at $1000 \mathrm{~Hz}$. The data recording was extended for 1 more minute after the task was done and the subjects relaxed themselves. Five datasets in total were collected for each subject.

\subsection{Simulation}

In order to verify the validation of the improved EMD scheme for mode mixing handling, the current study constructed 3 mixed signals with intermittent noises. Since the frequencies of brain waves fall into the range of $0.5-30 \mathrm{~Hz}$, the simulated signals were set in this range with high frequency intermittent noises.

(1) Set the sampling frequency at $1000 \mathrm{~Hz}$. Construct a $1000 \mathrm{~ms}$ sinusoid signal of amplitude $1 \mathrm{uV}$ at $3 \mathrm{~Hz}$, denote it as SO.

(2) Add 4 different $200 \mathrm{~Hz}$ intermittent signal of amplitudes $0.1,0.2,0.3$, and 0.4 at 4 random different places of the original signal, forming mixed signal S1. 
(3) In order to keep the generality, add $12 \mathrm{~Hz}$ and $12 \mathrm{~Hz}+25 \mathrm{~Hz}$ to S1 to form S2 and S3, respectively.

(4) Construct a matrix with S1, S2, and S3, apply SVD and GMM, eliminate insignificant features corresponding to small singular values and reconstruct the signals. Use EMD to decompose the reconstructed signals, compare the results with that of the direct application of EMD and EEMD.

The simulation results were as following:

(1)The original signal and 3 mixed signals are shown in Figure 2.

(2)through SVD and GMM clustering, the critical feature information in signals were accurately extracted. As shown in Figure 3, the red circles indicate singular values corresponding to significant features and blue ones indicate insignificant features (listed only 20 singular values).

(3) The decomposition results of EMD, EEMD and proposed scheme (Figure 4).

The simulation results suggest that the proposed feature extraction scheme have better performance than the traditional EMD and EEMD for removing mode mixing. Moreover, the spectrum analysis showed that the IMF components demonstrated better physical meaning. These results encouraged us to apply this method to actual EEG data analysis.

\subsection{Data analysis}

The following flowchart (Figure 5.) shows our scheme design, comparing with traditional method.

\subsubsection{Preprocessing}

Preprocessing is an important step affecting feature extraction and classification performance. In this work, filtering and artifacts removal were done in EEGLAB [30].

\subsubsection{Feature extraction}

The preprocessed signals were divided into $725 \mathrm{~ms}$ segments $(125 \mathrm{~ms}$ stimulation $+600 \mathrm{~ms}$ blank interval), feeding to SVD and GMM clustering. After the noise, artifacts, and insignificant features were filtered out, the EEG signals were reconstructed and decomposed via EMD.

An EEG signal $x(t)$ can be decomposed into $\mathrm{n}$ IMF components $c_{1}(t), c_{2}(t), \ldots c_{n}(t)$ and a residue res. If the residue is small enough to be ignored, then the total energy of the IMF components approximately equals to the energy of the original EEG signal, i.e., $E=\sum_{i=1}^{n} E_{i}$, where $E$ is the energy of the original signal, $E_{i}$ is the energy of the $i$-th IMF component. If we define $P_{i}=E_{i} / E$, then $P_{i}$ indicates the ratio of the energy of the $i$-th IMF component over the total energy. Therefore, we have the following definition:

$$
H=-\sum_{i=1}^{n} P_{i} \log P_{i} \square 1 \square
$$


Where $H$ stands for energy entropy.

After being decomposed by EMD, EEMD, and proposed scheme, the energy entropies of the IMFs mapping to $\delta, \theta, a$ and $\beta$ frequencies were extracted as the classification features.

\subsubsection{Classification}

SVM with RBF, linear, and polynomial kernel functions were used as the classifier. In this study, we trained 5 classifiers by using the 5 data set, each corresponding to one direction for each subject. An incoming unknown signal will pass through the 5 classifiers, and their votes will determine the category of the unknown EEG signal. Since the classification accuracy of each classifier was high enough, it is reasonable to expect their votes will give better performance. By feeding the extracted feature sets to SVM classifier and voting, the classification accuracies of P300 and that of a single trial EEG signal were achieved.

\section{Results}

\subsection{The classification results of P300 response and single trial EEG signals}

The classification accuracies of P300 responses by using SVM with EMD, EEMD, and our proposed scheme are shown in Figure 6. It is clear that the classification accuracies of P300 response with the proposed scheme $(96.6 \%, 96.7 \%$, and $96.1 \%$ with RBF, linear, and polynomial kernel functions, respectively) were higher than that of traditional EMD and EEMD. See Figure 6 for details.

A single trial consists of five consecutive flashes. The classification accuracies of single trial EEG signals with the proposed scheme $(85.0 \%, 85.5 \%$, and $81.8 \%$ with RBF, linear, and polynomial kernel functions, respectively) were significantly higher than that of traditional EMD and EEMD as well. See Figure 7. for details.

\subsection{The classification results with voting}

Through voting of SVM classifiers with any of the three types of kernel functions, the classification accuracies of P300 responses and single trial EEG signals were significantly improved, demonstrating the superiority and robustness of the voting mechanism. (The classification accuracies of P300 responses through voting were $98.5 \%, 98.7 \%$, and $98.0 \%$ for RBF, linear, and polynomial kernel functions, respectively. The improvements comparing with traditional EMD were $3.4 \%, 2.6 \%$, and $2.0 \%$, respectively; the improvements comparing with EEMD were $1.4 \%, 1.5 \%$, and $0.3 \%$, respectively. The classification accuracies of single trial EEG signals, i.e., the moving directions, were $92.4 \%, 93.6 \%$, and $90.4 \%$ for the three types of kernel functions, respectively. The improvements comparing with EMD were $14.9 \%, 11.4 \%$, and $8.9 \%$, respectively; the improvements comparing with EEMD were $6.1 \%, 6.8 \%$, and $1.4 \%$, respectively. See Figure 8 and Figure 9 for details.) 
To sum up, our results showed that: (1) IMF energy entropy is suitable feature for EEG signal classification with SVM classifier; (2) our proposed scheme can remove mode mixing more effectively than traditional EMD and EEMD and the decomposed IMFs were more physically meaningful; (3) the proposed voting mechanism significantly improved classification accuracy.

\section{Discussion}

The application of $\mathrm{BCl}$ is mainly limited by the classification accuracy, making the improvement of it critical. Therefore, the current study focused attention on improving the performance of single trial EEG signal classification scheme. The designed $\mathrm{BCI}$ was simple and effective, successfully eliciting P300 response. Through simulation we first verified the validation of the proposed scheme, and proved that IMF energy entropy was a suitable feature for single trial EEG signal classification. SVD and GMM were applied to remove the insignificant features. The vectors corresponding to the small singular values contain less information. If we assume that the noises had far less amplitudes than the signals (most likely, it is true for EEG signal processing), then it is safe to consider these vectors insignificant and remove them. The reconstructed EEG signals would be "cleaner", thus helping to solve the mode mixing problem caused by high frequency intermittent noises in the process of EMD. Some of the IMFs were strongly correlated to certain frequencies. Since the information of brain wave mainly concentrate in the $\delta, \theta, a$, and $\beta$ bands, we selected the corresponding IMFs, computed their energy entropies, and saved them as the feature sets. The improved classification results with SVM confirmed that SVD+GMM+EMD is an effective strategy for feature extraction. Since the classification accuracy of each SVM classifier for P300 responses was higher than 95\%, it is not surprising that the voting of a bunch of these classifiers will improve the performance. A total of 5 classifiers (one for each direction) were trained in the present study, their votes did improve the classification accuracies as expected.

There are still some unsolved issues in the process of data collection. The mental state of the subject cannot be effectively and accurately monitored, thus their concentration level cannot be measured and redressed directly, which reduces the occurrences of P300 responses and applicability of the $\mathrm{BCl}$. Moreover, the heavy computation load of SVD limited its performances for high dimensional data set. The prolonged processing time also limited the efficiency of the $\mathrm{BCl}$. A previous study reported that color heterogeneity may increase the attention level of the subject [31]; and Kouziokas proposed a new SVM kernel function which improved classification accuracy [32]. These results brought insights for our feature research. We should incorporate elements that improves subject's attention level into $\mathrm{BCl}$ design, further optimize the $\mathrm{BCl}$ and algorithm, so as to achieve faster processing speed and higher classification accuracy.

\section{Declarations}

Author contributions 
Song Luo: Conceptualization,Methodology,Writing-Original Draft preparation. PeiYun Zhong: Conceptualization,Methodology,Writing-Original Draft preparation. Rui Chen: Methodology. CunYang Pan: Data Curation,Validation. KeYu Liu: Data Curation,Validation. Kun Li: Supervision, Writing-Review \& Editing.All the authors have reviewed and approved the final manuscript.

\section{Acknowledgments}

This study was funded by the Guizhou Provincial Science and Technology Foundation ([2016]1412 and [2017]1418), the Innovation Project Funding for Chinese Overseas Returnees [2016]25, and the Guizhou Normal University Doctoral Start Up Fund for Kun Li[2018].

\section{Conflict of interests}

The authors declare no conflict of interests.

\section{References}

[1]Greely, H., Ramos, K.\& Grady, C. Neuroethics in the Age of Brain Projects, Neuron. 92(2016)637-641. https://doi.org/10.1016/j.neuron.2016.10.048.

[2]Okano, H., Miyawaki, A.\& Kasai, K. Brain/MINDS: brain-mapping project in Japan, Philos. Trans. R. Soc. Lond. B. Biol. Sci. 370(2015)1688. https://doi.org/10.1098/rstb.2014.0310.

[3]Okano, H. et al. Brain/MINDS: A Japanese National Brain Project for Marmoset Neuroscience, Neuron. 92(2016)582-590. https://doi.org/10.1016/j.neuron.2016.10.018.

[4]Poo, M., Du, J., Xiong, Z., Xu, B.\& Tan, T. China Brain Project: Basic Neuroscience, Brain Diseases, and Brain-Inspired Computing, Neuron. 92(2016)591-596. https://doi.org/10.1016/j.neuron.2016.10.050.

[5]Arbel, Y., Mccarty, K. N., Goldman, M., Donchin, E.\& Brumback, T. Developmental changes in the feedback related negativity from 8 to 14years, Int. J. Psychophysiol. 132(2018)331-337. https://doi.org/10.1016/j.ijpsycho.2018.01.004.

[6]Fu, Y. et al. Single-trial decoding of imagined grip force parameters involving the right or left hand based on movement-related cortical potentials, Chinese. Sci. Bull. 59(2014)1907-1916. https://doi.org/10.1007/s11434-014-0234-5.

[7]Chaudhary, S., Taran, S., Bajaj, V.\& Siuly, S. A flexible analytic wavelet transform based approach for motor-imagery tasks classification in BCl applications, Comput. Meth. Pro. Bio. 187(2020)105325. https://doi.org/10.1016/j.cmpb.2020.105325.

[8]Huang, N. E. et al. The empirical mode decomposition and the Hilbert spectrum for nonlinear and nonstationary time series analysis, P. Roy. Soc. A-MATH. Phy. 454(1998)903-995. https://doi.org/10.1098/rspa.1998.0193. 
[9]Kevric, J.\& Subasi, A. Comparison of signal decomposition methods in classification of EEG signals for motor-imagery BCl system, Biomed. Signal. Proces. 31(2017)398-406.

https://doi.org/10.1016/j.bspc.2016.09.007.

[10]Taran, S., Bajaj, V., Sharma, D., Siuly, S.\& Sengur, A. Features based on analytic IMF for classifying motor imagery EEG signals in BCl applications, Measurement 116(2017)68-76.

https://doi.org/10.1016/j.measurement.2017.10.067.

[11]Tang, X., Li, W., Li, X., Ma, W.\& Dang, X. Motor imagery EEG recognition based on conditional optimization empirical mode decomposition and multi-scale convolutional neural network, Expert. Syst. Appl. 149(2020)113285. https://doi.org/10.1016/j.eswa.2020.113285.

[12]Popovych, S. et al. Movement-related phase locking in the delta-theta frequency band, Neuroimage 139(2016)439-449. https://doi.org/10.1016/j.neuroimage.2016.06.052.

[13]Crovato César, C. D.\& Schuck, A. The use of wavelet packet transform and artificial neural networks in analysis and classification of dysphonic voices, IEEE. Trans. Biomed. Eng. 54(2007)1898-1900. https://doi.org/10.1109/TBME.2006.889780.

[14] Zhang, C., Zheng, C.\& Yu, X. Automatic recognition of cognitive fatigue from physiological indices by using wavelet packet transform and kernel learning algorithms, Expert. Syst. Appl. 36(2009)4664-4671. https://doi.org/10.1016/j.eswa.2008.06.022.

[15]Arjmandi, M. K.\& Pooyan, M. An optimum algorithm in pathological voice quality assessment using wavelet-packet-based features, linear discriminant analysis and support vector machine, Biomed. Signal. Proces. 7(2012)3-19. https://doi.org/10.1016/j.bspc.2011.03.010.

[16]Wu, Z. N.\& Huang, E. ENSEMBLE EMPIRICAL MODE DECOMPOSITION: A NOISE-ASSISTED DATA ANALYSIS METHOD, Adv. Adaptive. Data. Analysis. 1(2009)1-41.

https://doi.org/10.1142/S1793536909000047.

[17]Huang, N.E., Shen, Z.\& Long, S.R. A NEW VIEW OF NONLINEAR WATER WAVES: The Hilbert Spectrum1, Annu. Rev. Fluid. Mech. 31(1999)417-457. https://doi.org/10.1146/ANNUREV.FLUID.31.1.417.

[18]Huang, H., Wei, S., Chao, H., Chang, F.\& Chi, S. An Investigation Study on Mode Mixing Separation in Empirical Mode Decomposition, IEEE. Access. 99(2019). https://doi.org/10.1109/ACCESS.2019.2930543.

[19]Chen, X., Lin, J., Huang, C.\& He, L. A novel method based on Adaptive Periodic Segment Matrix and Singular Value Decomposition for removing EMG artifact in ECG signal, Biomed. Signal.

Proces. 62(2020)102060. https://doi.org/10.1016/j.bspc.2020.102060.

[20]Hyunjoong, K., Han, K. K.\& Sungzoon, C. Improving spherical k-means for document clustering: Fast initialization, sparse centroid projection, and efficient cluster labeling, Expert. Syst.

Appl. 150(2020)113288. https://doi.org/10.1016/j.eswa.2020.113288. 
[21]Nowakowska, E., Koronacki, J.\& Lipovetsky, S. Clusterability assessment for Gaussian mixture models, Appl. Math. Comput. 256(2015) 591-601. https://doi.org/10.1016/j.amc.2014.12.038.

[22]Fu, Y., Liu, X., Sarkar, S.\& Wu, T. Gaussian Mixture Model with Feature Selection: An Embedded Approach, Comput. Ind. Eng. 152(2020) 107000. https://doi.org/10.1016/J.CIE.2020.107000.

[23]Raghu, S., Sriraam, N.\& Kumar, G. P. Classification of epileptic seizures using wavelet packet log energy and norm entropies with recurrent Elman neural network classifier, Cognitive.

Neurodynamics. 11(2017)1-16. https://doi.org/10.1007/s11571-016-9408-y.

[24]Göksu, H. BCl oriented EEG analysis using log energy entropy of wavelet packets, Biomed. Signal. Proces. 44(2018)101-109. https://doi.org/10.1016/j.bspc.2018.04.002.

[25]Siuly, Yan, L.\& Wen, P. Clustering technique-based least square support vector machine for EEG signal classification, Comput. Meth. Prog. Bio. 104(2011)358-372. https://doi.org/10.1016/j.cmpb.2010.11.014.

[26]Al-Hadeethi, H., Abdulla, S., Diykh, M., Deo, R. C.\& Green, J. H. Adaptive boost LS-SVM classification approach for time-series signal classification in epileptic seizure diagnosis applications, Expert. Syst. Appl. 161(2020)113676. https://doi.org/10.1016/J.ESWA.2020.113676.

[27]Asadi, M., Mohammad, A. J. J., Saeed, P.\& Vahid, M. Detecting botnet by using particle swarm optimization algorithm based on voting system, Future. Gener. Comp. Sy. 107(2020)95-111. https://doi.org/10.1016/j.future.2020.01.055.

[28]García-Gutiérrez, J., Mateos-García, D., Garcia, M.\& Riquelme-Santos, J. C. An evolutionary -weighted majority voting and support vector machines applied to contextual classification of LiDAR and imagery data fusion, Neurocomputing, 163(2015)17-24. https://doi.org/10.1016/j.neucom.2014.08.086.

[29]Jongsma, M. L. A. et al. The learning-oddball paradigm: Data of 24 separate individuals illustrate its potential usefulness as a new clinical tool, Clin. Neurophysiol. 124(2013)514-521.

https://doi.org/10.1016/j.clinph.2012.09.009.

[30]Maren, S., Anna-Katharina, R. B., Stefan, D.\& Martin, G. B. Source-Modeling Auditory Processes of EEG Data Using EEGLAB and Brainstorm, Front. Neurosci. Switz. 12(2018)1-11.

https://doi.org/10.3389/fnins.2018.00309.

[31]Fernandez-Rodriguez, A., Velasco-Álvarez, F., Medina-Juliá, M. T.\& Ron-Angevin, R. Evaluation of flashing stimuli shape and colour heterogeneity using a P300 brain-computer interface speller, Neurosci Lett. 709(2019)134385. https://doi.org/10.1016/j.neulet.2019.134385.

[32]Kouziokas, G. N. SVM kernel based on particle swarm optimized vector and Bayesian optimized SVM in atmospheric particulate matter forecasting, Appl. Soft. Comput. 93(2020)106410.

https://doi.org/10.1016/j.asoc.2020.106410. 
Figures

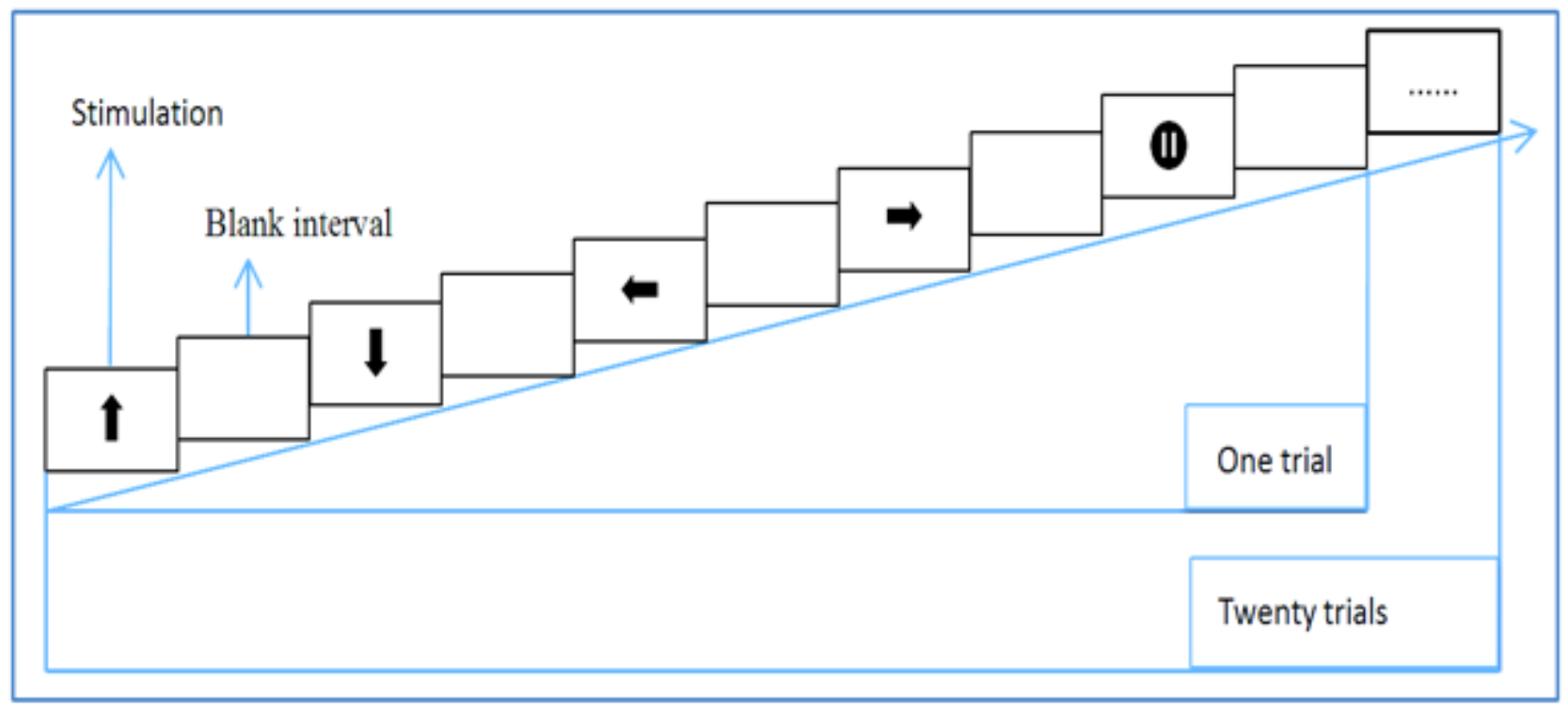

Figure 1

The stimulation sequence and time windows
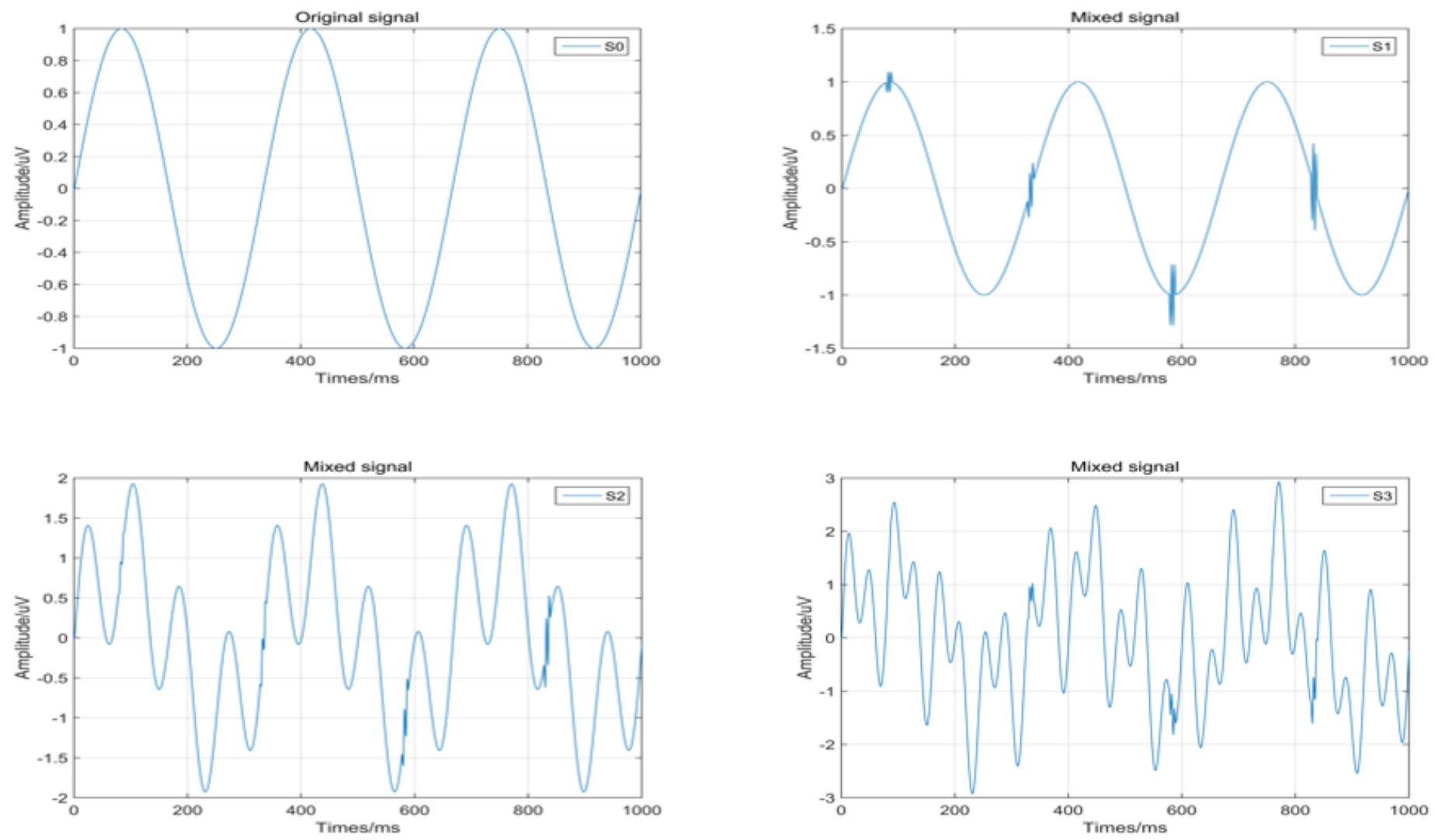

Figure 2 
Original signal and 3 mixed signals
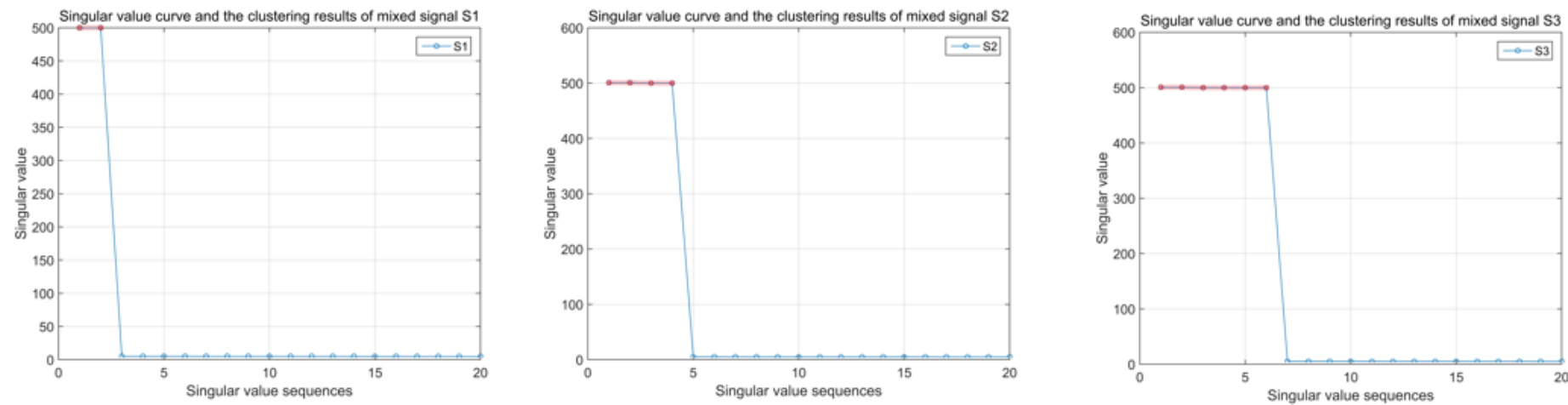

Figure 3

The SVD and GMM clustering results of the 3 mixed signals

Signal S1

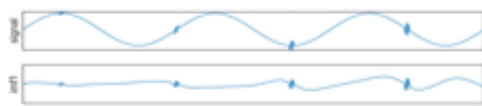

EMD
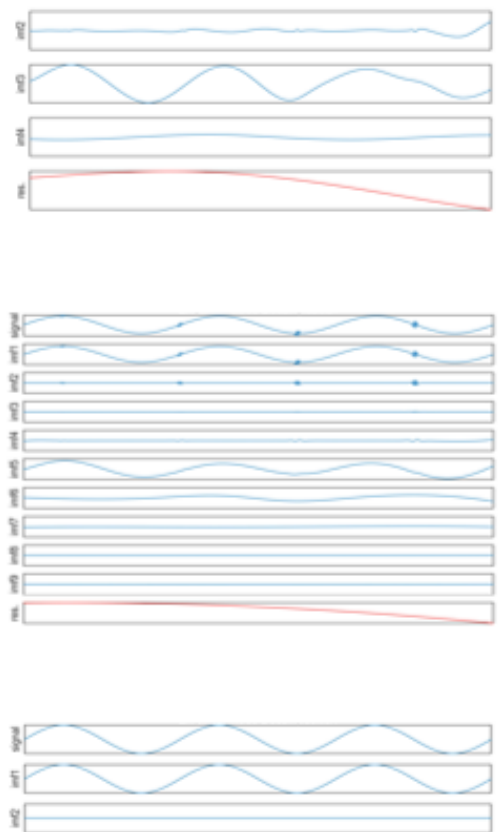

\section{Proposed}

scheme

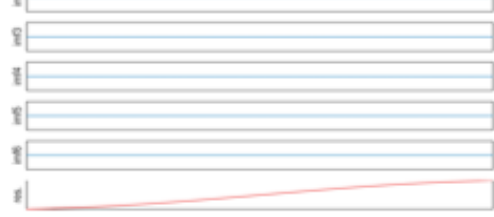

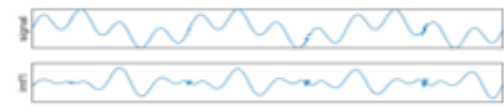
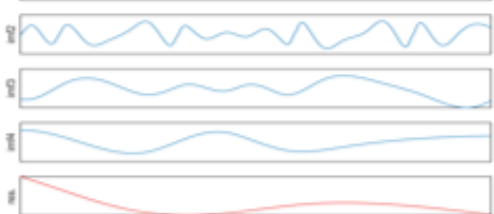

Signal S2

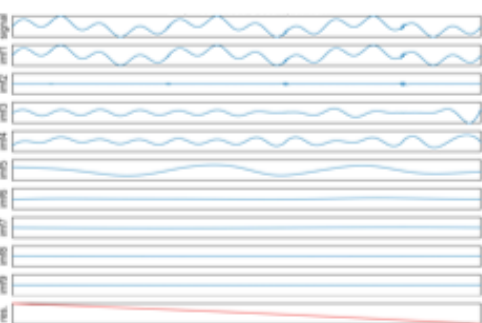

\section{Signal S3}
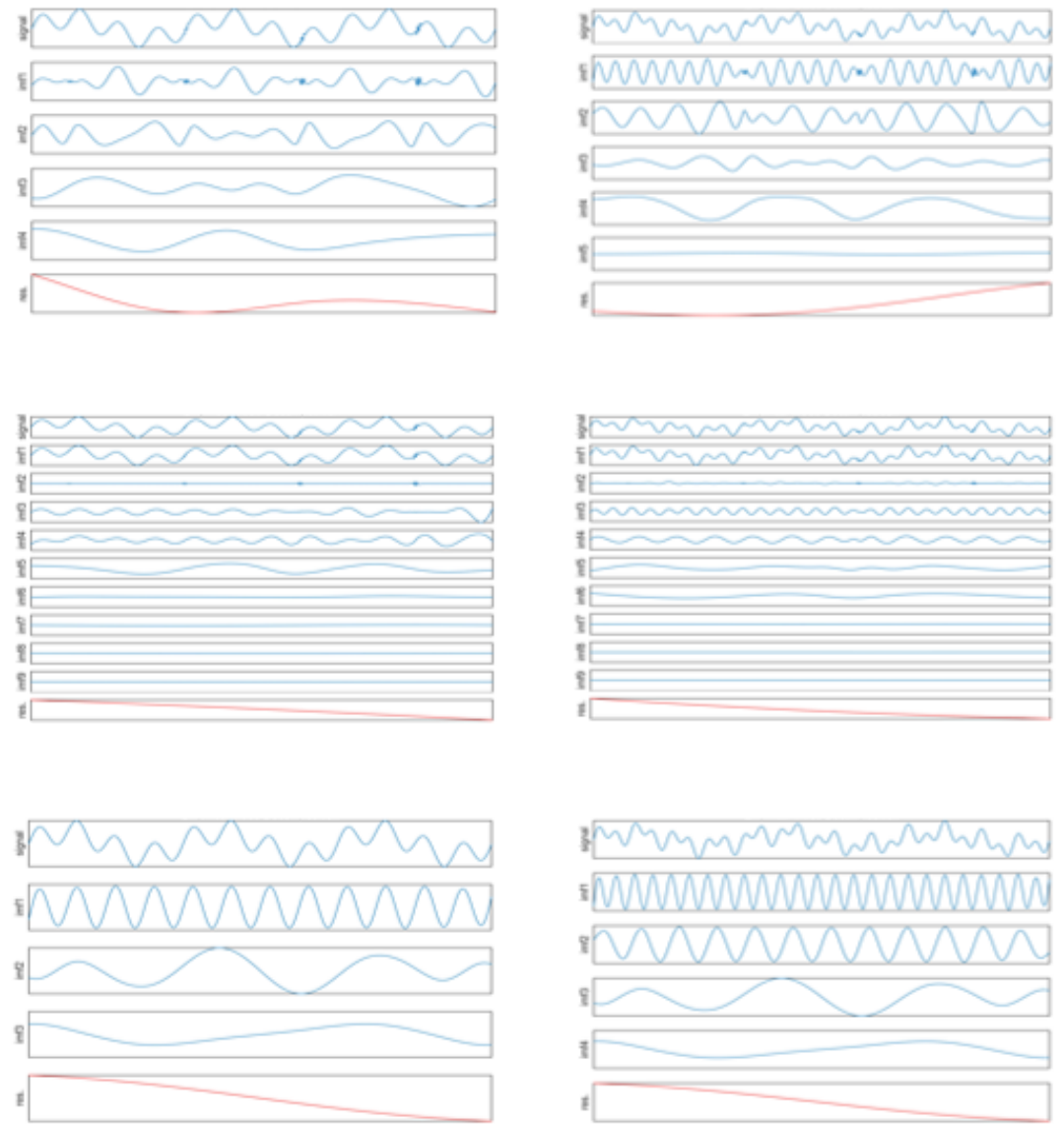

\section{Figure 4}

The decomposition results of the 3 mixed signals by EMD, EEMD and the proposed scheme 


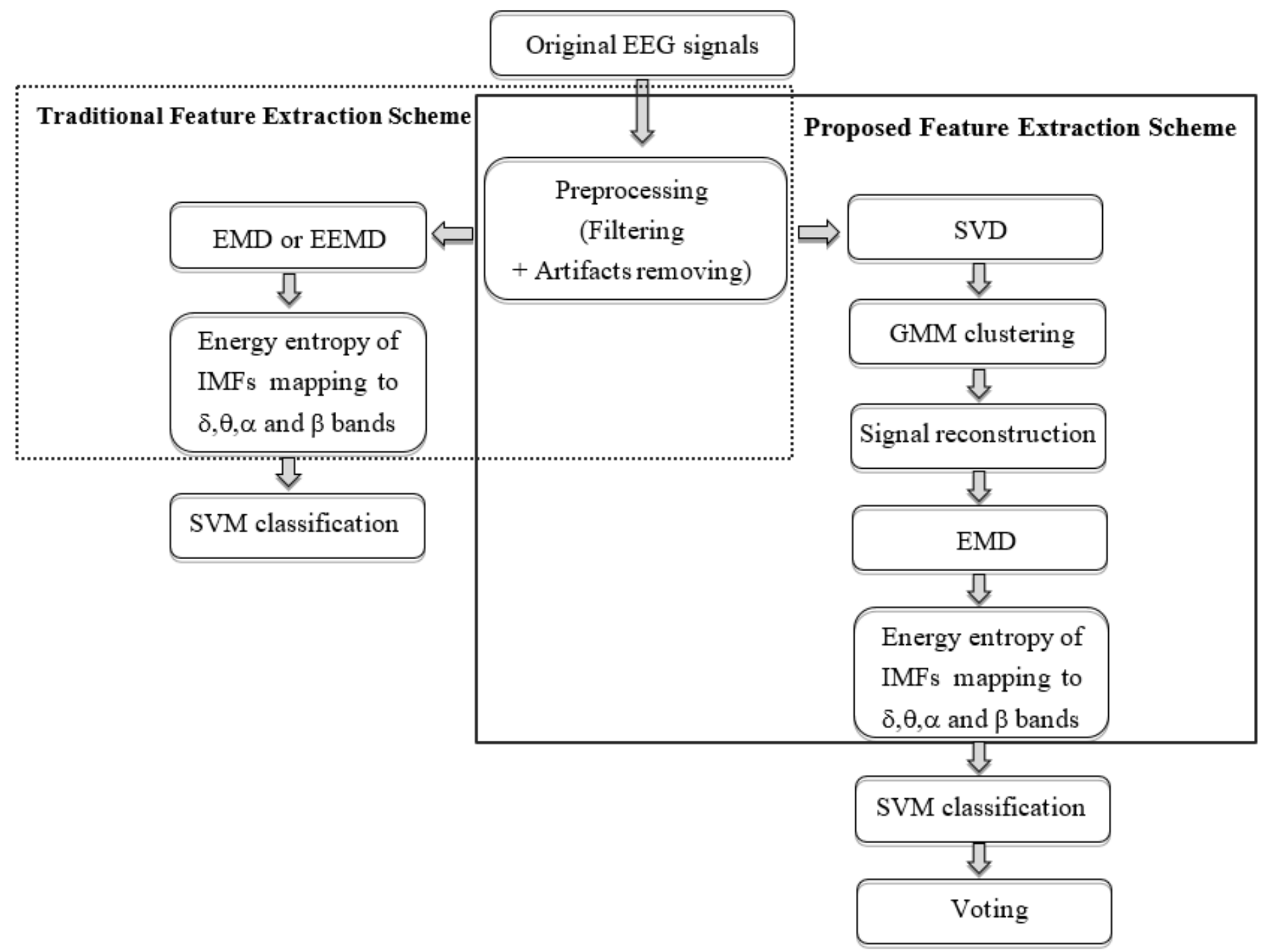

Figure 5

The flowchart of data analysis 


\section{Classification accuracies of $\mathrm{P300}$ response (based on SVM)}

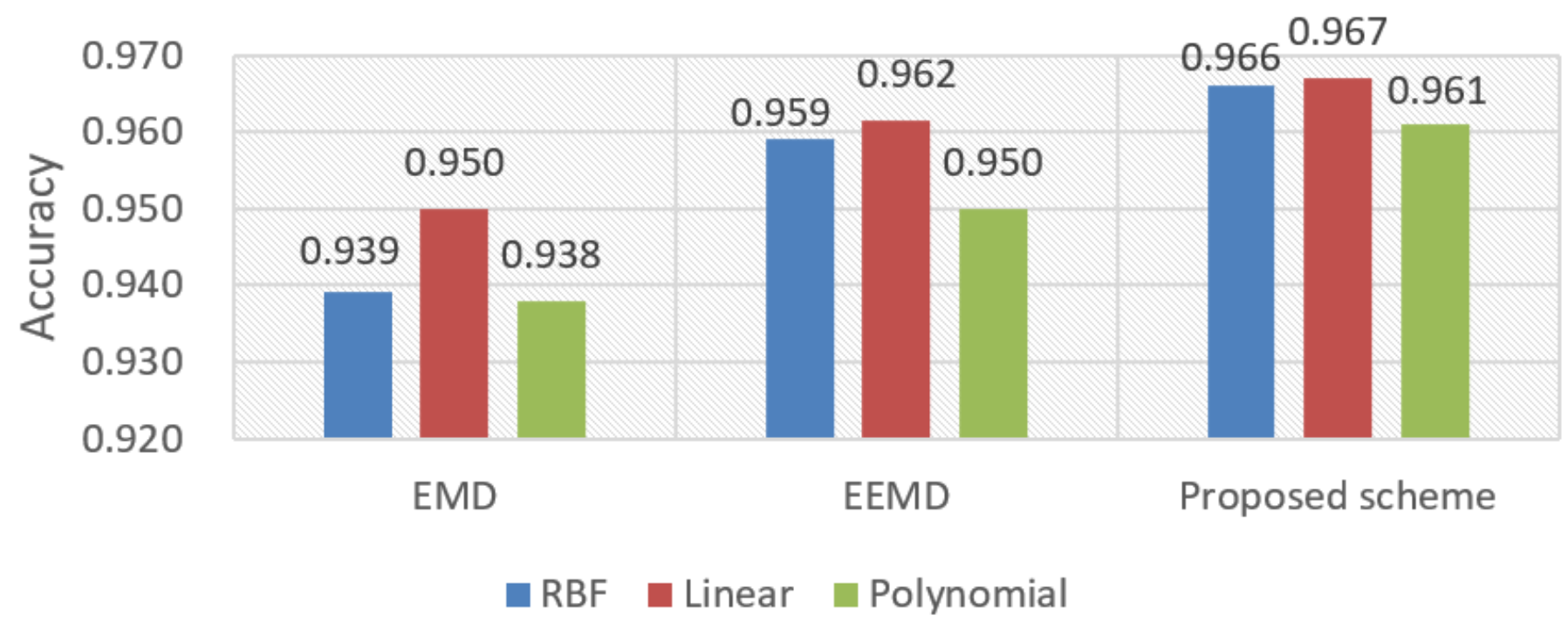

Figure 6

Comparison of P300 response classification accuracies by using SVM classifiers with EMD, EEMD, and our proposed scheme

\section{Classification accuracies of single trial EEG signal ( based on SVM)}
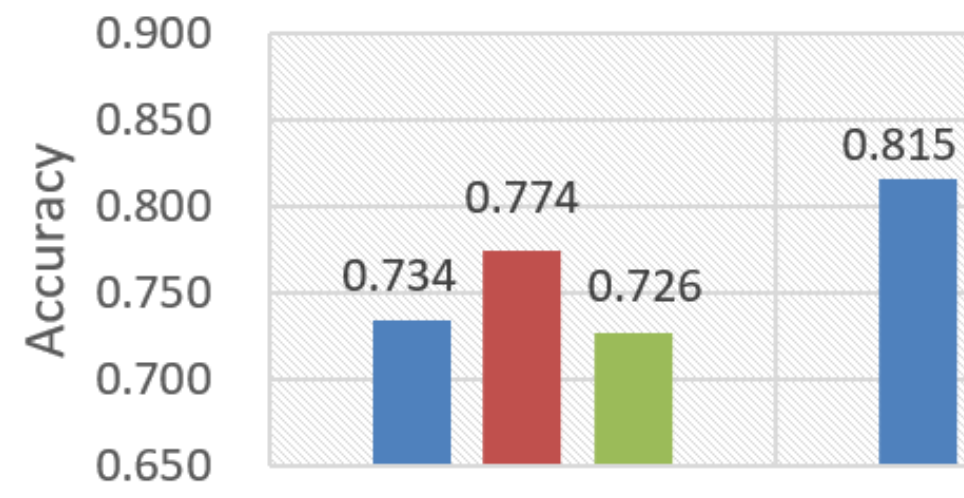

EMD

EEMD

Proposed scheme

nBF Linear Polynomial 
Comparison of the classification accuracies of single trial EEG signals by using SVM with EMD, EEMD, and our proposed scheme

\section{Classification accuracies of P300 response (based on Voting)}

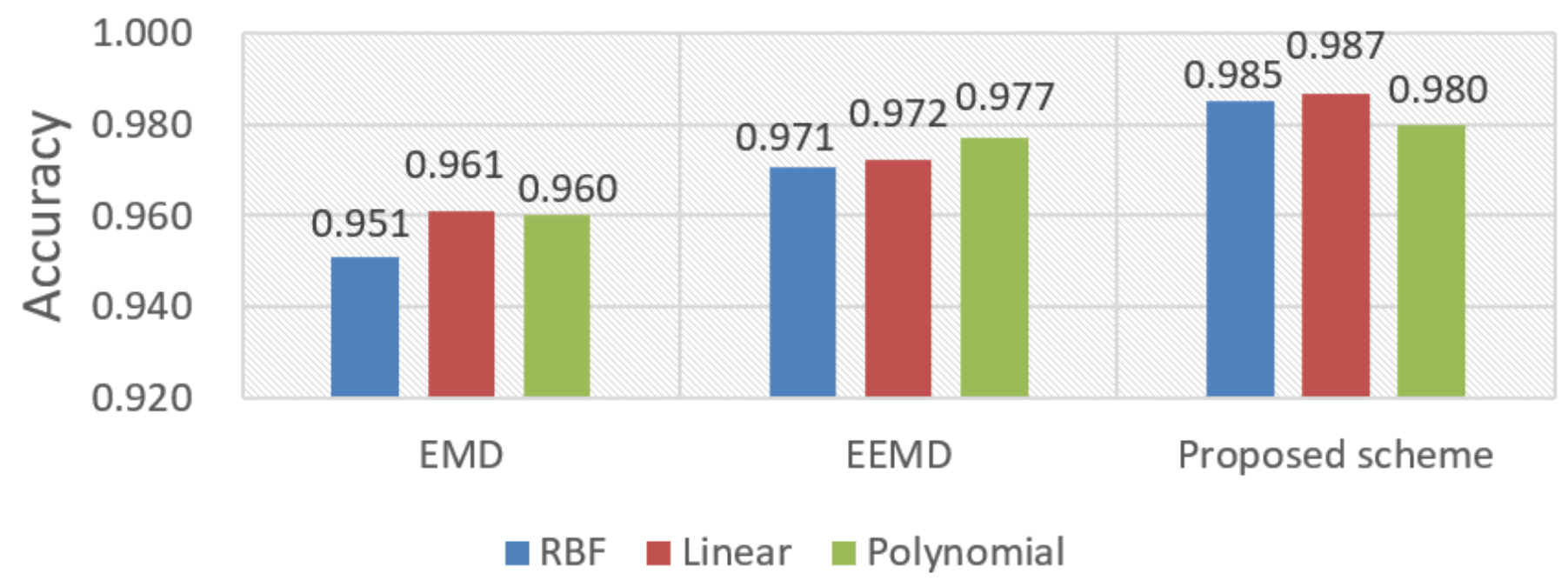

Figure 8

Comparison of P300 response classification accuracies through voting

\section{Classification accuracies of single trial EEG signals (based on Voting)}
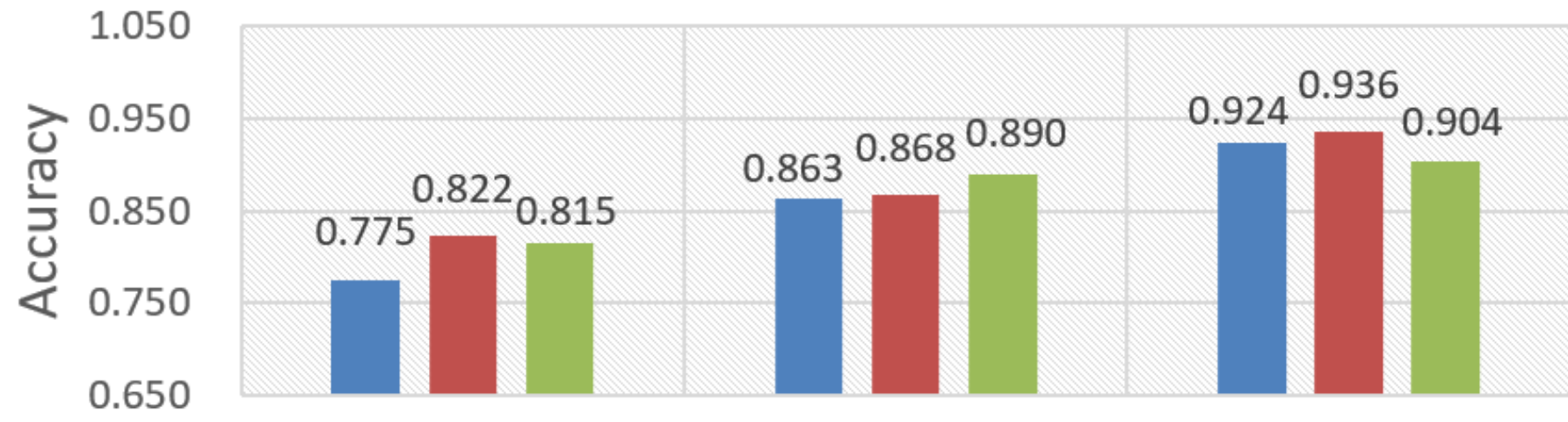

EMD

EEMD

Proposed scheme

- RBF Linear $\quad$ Polynomial 
Comparison of single trial EEG signal classification accuracies through voting 